\title{
The A20/TNFAIP3-CDC20-CASP1 Axis Promotes Inflammation-mediated Metastatic Disease in Triple-negative Breast Cancer
}

\author{
CHRISTINE SONG ${ }^{1,2}$, AYSE TUBA KENDI $^{1}$, VAL J. LOWE ${ }^{1}$ and SEUNGBAEK LEE ${ }^{1,3}$ \\ ${ }^{1}$ Division of Radiology, Mayo Clinic, Rochester, MN, U.S.A.; \\ ${ }^{2}$ Mayo High School, Rochester, MN, U.S.A.; \\ ${ }^{3}$ Department of Molecular Pharmacology and Experimental Therapeutics, Mayo Clinic, Rochester, MN, U.S.A.
}

\begin{abstract}
Background/Aim: The functions of the specific genes involved in the three types of breast cancer $(B C)$ are unclear. Materials and Methods: A total of 53,805 genes were assessed from the RNA-sequencing database of $B C$ cells and classified into those involved in hormonal positive $(H R+) B C$ and triple-negative breast cancer (TNBC). Overall, distant metastasis-free, and relapse-free survival obtained from the Breast Cancer Gene-Expression Miner database containing 13,603 human breast cancer patient samples were assessed for gene associations using the RNAsequencing database. To examine cell invasion and cytokine levels, inflammation-related genes were knocked down. The role of inflammation in cancer metastasis was confirmed using inflammatory inhibitors in a three-dimensional organoid ex vivo. Results: Genes affecting inflammation and cancer metastasis were highly expressed in TNBC, unlike $H R+B C$. The A20/TNFAIP3-CDC20-CASP1 axis, which includes inflammation-related genes found in TNBC, was associated with poor patient prognosis, cancer metastasis, and cytokine levels. Inflammation inhibitors prevented the metastasis of aggressive TNBC. Conclusion: The A20/TNFAIP3-CDC20-CASP1 axis is closely related to the metastatic potential of TNBC, and inflammation inhibitors might be a novel target therapy for TNBC.
\end{abstract}

This article is freely accessible online.

Correspondence to: Val J. Lowe, Division of Radiology, Mayo Clinic, 200 First Street SW, Rochester, MN 55905, U.S.A. Tel: +1 5072849599, e-mail: vlowe@mayo.edu; SeungBaek Lee, Division of Radiology, Mayo Clinic, 200 First Street SW, Rochester, MN 55905, U.S.A. Tel: +1 5077228654, e-mail: lee.seungbaek@mayo.edu

Key Words: Triple-negative breast cancer (TNBC), A20/TNFAIP3, cell division cycle 20 (Cdc20), CASP1/Caspase-1, inflammation, metastasis, inflammation inhibitors.
Breast cancer is the leading cause of women's cancer death and the second most common cancer worldwide (1-6). One subtype of breast cancer is characterized by the expression of hormone receptors (estrogen and progesterone receptors) and ERBB2 (also known as HER2) over-expression and/or amplification, and these receptors are the main targets of effective target therapies $(1,2,6)$. Another subtype, triplenegative breast cancer (TNBC), lacks expression of all of these receptor proteins and accounts for about $10-15 \%$ of all breast cancers (1-3). As hormonal therapy targeting estrogen receptors or drug treatment targeting HER2 is fruitless in this group, systemic treatment with chemotherapy remains the only treatment option $(1,3,5,6)$. Also, TNBC is the most aggressive subtype that easily metastasizes to other organs $(1-3,5)$. Although TNBC usually responds well to initial chemotherapy, it tends to recur more often than other breast cancers (1-3). More research on TNBC is required and studies on the relationship between genes and TNBC are still lacking. Therefore, the characterization of metastasis-related genes is important to improve the current lack of therapeutic options and increase the survival rates of TNBC patients.

Inflammation is often closely related to the formation and progression of cancer (7-10). Tumor-associated inflammatory responses are triggered by many external factors, including bacterial and viral intracellular infections, autoimmune diseases, obesity, tobacco smoking, exposure to cancercausing chemicals, and excessive alcohol consumption (7-9). This leads to a disproportionate inflammatory response, which ultimately promotes cancer formation and malignant progression (7-9). Mutations in genes that suppress inflammation in the body lead to the abnormal activation of inflammation in cells, which may lead to malignancy. Ultimately, both extrinsic inflammation, which promotes inflammatory conditions that predispose to cancer, and intrinsic inflammation, which is caused by genetic events that cause neoplasia, are important factors in tumor development by suppressing the body's immune system $(7-9,11)$. There are 
several inflammatory factors that induce cancer such as proinflammatory cytokines, tumor necrosis factor (TNF- $\alpha$ ), interleukins $1 \alpha$ (IL-1 $\alpha$ ), IL-1 $\beta$, IL-6, IL-8, IL-18, and chemokines $(9,12-14)$. A20/TNFAIP3 is a well-known key regulator of inflammation and immunity that has a role in inhibiting the NF-kB signaling pathway and apoptosis $(4,10$, 15). Several recent reports have suggested an oncogenic role of A20/TNFAIP3 in various solid tumor cell lines $(4,10,15-$ 18). A20/TNFAIP3 is significantly up-regulated in TNBC and its expression level is highly correlated with low survival of metastasis-free patients, and promotes cancer metastasis via multi-monoubiquitylation, which activates the functions of Snail (4). Therefore, the role of A20/TNFAIP3 in the inflammatory response and carcinogenesis remains unclear. Also, Caspase-1 has been reported to regulate proinflammatory cytokine IL-1 $\beta$ and IL-18 (12-14).

Cell division cycle $20(\mathrm{Cdc} 20)$ is a key anaphasepromoting complex or cyclosome $(\mathrm{APC} / \mathrm{C})$ activator that is also a mitosis-related oncogene/oncoprotein (2, 19-21). Most of the $\mathrm{Cdc} 20$ or Cdh1 substrates are regulators that play a major role in cell division, such as Cyclin A, Cyclin B (for mitotic cyclins), Aurora A, Aurora B, Plk1, Nek2A (for mitotic kinases), Securin, Sgo1 (for chromosome segregation), Survivin (for mitotic checkpoint), Geminin, Cdc6 (DNA replication), Skp2 (F box for S phase), and Ets2 and FoxM1 (for transcription) (2, 19-23). Cdc20 is not expressed or is expressed at very low levels in G1, but when cells enter division, $\mathrm{Cdc} 20$ binds to $\mathrm{APC} / \mathrm{C}$ or Parkin complex and acts as a major regulator through substrate ubiquitination $(19,24,25)$. The main period of Cdc20 action is from Nuclear Envelope Breakdown (NEBD) to anaphase, in which daughter chromosomes are formed. Cdc20 also functions as a mitotic checkpoint (also known as the spindle assembly checkpoint) by localizing and binding to kinetochore proteins such as Mad2, Bub3, and BubR1 (1923). It is converted to the final mitotic checkpoint inhibitor Bub3-BubR1-Cdc20, which blocks APC/C-dependent ubiquitination, stabilizing cyclin $\mathrm{B}$ and securin and preventing progression to anaphase $(20,21,23)$. Excessively high expression of $\mathrm{Cdc} 20$ promotes cell division and is closely related to various types of human cancers such as breast, osteosarcoma, glioblastoma, pancreatic, hepatocellular, prostate, colorectal, lung, bladder, and liver cancer $(2,19,22,23)$. Especially, over-expression of Cdc20 has been associated with an aggressive course of breast cancer. Recently, our group reported that the CDC20 gene is highly expressed in three TNBC cell lines and 10,001 breast cancer patients tissue samples $(2,22,23)$. We confirmed that high Cdc20 expression is also associated with poor clinical outcomes such as low overall, distant-metastasis free, and relapse-free survival in TNBC patients and loss of the CDC20 gene decreases tumor cell growth and migration in human TNBC cell line, MDA-MB-231 (2).
The three-dimensional (3D) organoid ex vivo technique is an advanced cell culture method that overcomes the limitations of general 2D cell culture and animal experiments (26-30). When cancer cells are cultured for a long time in a 2D cell culture, they lose their unique properties and characteristics, and the effects of drugs are different from those of the tumor cells $(27,31,32)$. In addition, the use of animals such as mice, rats, rabbits, cats, and dogs, which have many genetic differences from humans, also frequently result in erroneous anticancer effects $(26,27,31,32)$. 3D organoid culture decreases the failure rate of new drugs, and allows the examination of the effect of the drug directly on the actual human tissue and a more accurate determination of the anti-cancer effect of new drugs (27-31).

In this study, we assessed gene expression differences between hormonal positive breast cancer and TNBC through gene association experiments. We found that the expression of cell invasion and inflammation-related genes in TNBC is characteristically different from that of genes expressed in hormonal positive breast cancer. Especially, high A20/TNFAIP3, CDC20, and CASP1 expression in TNBC associates with poor clinical outcomes in breast cancer patients. We also found that inflammation-related genes, A20/TNFAIP3, and CASP1 show high correlation with $C D C 20$ in TNBC and positively regulate cancer cell invasion and inflammation-related cytokines. We confirmed that several inflammation inhibitors strongly prevented the metastasis of aggressive TNBC in cell culture experiments.

\section{Materials and Methods}

Cancer data collection and processing. To investigate gene expression in six different breast cancer cell lines, several cancer datasets from the Gene Expression Omnibus, containing patients' clinical information and gene expression data, were retrieved. Among the various datasets, we identified which genes are significantly over-expressed in TNBC human cell lines and TNBC patients. Briefly, a classification rule based on summarizing the standardized expression levels of genes was defined. First, the data from NCBI, including data regarding major mitosis-related genes and $\beta$-actin were selected. The variability of gene expression in human TNBC cell lines and TNBC patients was calculated by other independent research groups that used $\beta$-actin as the most stable housekeeping gene for normalizing gene expression. After each gene was normalized by $\beta$-actin, the individual gene values were determined. Then, the calculated values were divided by the sum of the averages of all genes. Finally, $\log 2$-transformed expression (or concentration) values were determined. Statistical analyses were performed by using the GraphPad Prism software (version 6.0, San Diego, CA, USA). For analyzing gene correlation to specific cellular processes such as cell metabolism, inflammation, or cancer metastasis, we used the DAVID database (2). The names of the 26 genes highly expressed in hormonal positive breast cancer were searched among the 53,805 genes of the DAVID database (containing data for six different breast cancer cell lines) for correlations (2). The 48 genes highly expressed in TNBC were then 
added to the DAVID database to analyze the correlation between the genes and specific classifications. The correlation between $C d c 20$ and other genes from the 53,805 genes dataset, was assessed using the Breast Cancer Gene-Expression Miner v4.5 (2). From this experiment, the correlation between the genes in ER+, ER-, HER2+, and TNBC subtypes was assessed. For determining the relationship between $C d c 20$, TNFAIP3/A20, and CASP1 with overall, distant metastasis-free, and relapse-free survival rates, the Breast Cancer Gene-Expression Miner v4.5 was used with human breast cancer patient dataset (2). $p$-Values were calculated using unpaired, twotailed Student's $t$-tests

Cells, cell lines and reagents. All cell lines were obtained from commercial venders such as ATCC (Manassas, VA, USA) (2). Human breast cancer cell lines were cultured in Dulbecco's modified Eagle's media (DMEM, Thermo Fisher Scientific, Waltham, MA, USA) (MDA-MB-435, MCF7, HS578T and MDAMB-231) or RPMI (ZR-75B, ZR-75-1, SK-BR-3, T47D, and BT549) (2). Normal breast cells, MCF-10A, were cultured in DMEM/Ham's F-12 nutrient mixture (GIBCO) with 5\% horse serum (GIBCO), $20 \mathrm{ng} / \mathrm{ml}$ EGF (Peprotech, East Windsor, NJ, USA), $10 \mu \mathrm{g} / \mathrm{ml}$ insulin, $0.5 \mu \mathrm{g} / \mathrm{ml}$ hydrocortisone (Sigma, St. Louis, MO, USA), and $100 \mathrm{ng} / \mathrm{ml}$ cholera toxin. Necrostatin-1 (RIPK1 inhibitor), Necrosulfonamide (NSA), and GW806742X (MLKL inhibitor) were purchased from Calbiochem (San Diego, CA, USA) or Sigma. The HA-Cdc20 plasmid was purchased from addgene (Watertown, MA, USA)

Gene silencing by lentiviral shRNAs. CDC20 lentiviral shRNAs (2, 19 ) and the negative Control shRNA were purchased from SigmaAldrich. A20/TNFAIP3 (sc-37655-V) or CSAP-1/Caspase-1 (sc29235-V) shRNAs human lentiviral particles were purchased from Santa Cruz Biotechnology (Dallas, TX, USA). MDA-MB-231 cells were infected or transfected with Control or $C D C 20$ shRNAs using Lipofectamine 2000 (Invitrogen, Waltham, MA, USA). To confirm gene knockdown, CDC20 protein expression levels were analyzed by western blot $(2,19)$.

Western blot analysis and antibodies. Protein lysate samples from different breast cancer cell lines were lysed by RIPA lysis buffer supplemented with protease inhibitors, including with $1 \mathrm{mM}$ PMSF on ice. Protein lysate samples were first separated using gel electrophoresis and then transferred onto PVDF membranes. The membranes were then blocked in TBS-T buffer containing 5\% fatfree milk for $1 \mathrm{~h}$ at room temperature. Next, the PVDF membrane was incubated with specific primary antibodies. Mouse monoclonal antibodies recognizing Cdc20 (sc-5296) and Caspase-1 (sc-56036) were purchased from Santa Cruz Biotechnology. Rabbit polyclonal antibody recognizing A20/TNFAIP3 (\#4625) was obtained from Cell Signaling Technology (Danvers, MA, USA). Anti- $\beta$-actin mouse antibody was purchased from Sigma. The membrane was washed three times with $1 \%$ TBST and subsequently incubated for $1 \mathrm{~h}$ with rabbit anti-mouse IgG-HRP secondary antibodies (Abcam, Cambridge, UK) as described previously (7, 19, 28, 29, 33).

Migration assays. For migration assays (wound healing assays) (33), cells were infected or transfected with shRNAs or DNA plasmids. Cells were seeded in 6 -well plates at a density of $1 \sim 3 \times 10^{3}$ cells/well, grown until confluence (around 2-3 days), and then the cells were refed with serum-free media for $24 \mathrm{~h}$. Confluent cells on a monolayer were scraped with a P200 tip in each well (3 lines/well), and the medium was replaced with complete medium. After $24 \sim 48 \mathrm{~h}$, the cells were fixed with $3.7 \%$ paraformaldehyde (Sigma) and stained with $0.2 \%$ crystal violet (Sigma). Images were obtained at 0,1 , and 2 days. Cell migration was quantified by counting the number of cells inside the scratch (at time 0 as standard) in each of the three different fields of the wound.

Invasion assays. For matrigel invasion assays (33), cells were infected with shRNAs in $10 \mathrm{~cm}$ tissue culture dishes. A total of 2,000 to 3,000 cells were seeded in a 24-well invasion chamber (Corning, 354480, Corning, NY, USA). After $18 \mathrm{~h}$, the medium was changed to serum-free medium, and cells grew until $\sim 80 \%$ confluence. The media were changed with media containing $20 \%$ FBS to create a cellular environment with high nutrition in the bottom chamber. To measure cell invasion after $24 \mathrm{~h}$, the filter was stained with $0.2 \%$ Crystal Violet and invaded cells were counted through a microscope. Each sample was plated in triplicate $(50,000$ cells/insert) to increase the validity of the experiment.

Primers and real-time polymerase chain reaction ( $q R T-P C R)$ with reverse transcription. RNA preparation, $\mathrm{CDNA}$ and $\mathrm{qRT}-\mathrm{PCR}$ were performed as described previously $(7,19)$. The following primers were used for real-time quantitative reverse transcription PCR (qRT-PCR).

TNF- $\alpha$ primer sequences: Forward 5'-CCAGCCAGCAGAAGCT CCCTCAGCGAG-3', Reverse 5'-GCGGATCATGCTTTCTGTGC TCATGGTGTC-3'; IL-6 primer sequences: Forward 5'CCTGCGTTTAAATAACATCAGCTTTAGCTT-3', Reverse 5'GCACAATGTGACGTCGTTTAGCATCGAA-3'; $I L-1 \beta$ primer sequences: Forward 5'-CACAGCAGCACATCAACAAG-3', Reverse 5'-GTGCTCATGTCCTCATCCTG-3'.

$3 D$ organoid assay. For 3D organoid assays, human breast cancer cell lines were cultured on NanoCulture plates (Scivax, Kawasaki, Japan). After seeding for 1 day, cells were infected with shRNAs or treated with inflammation inhibitors and then cells were observed at 7 days. Cell sphere or actin fiber numbers were observed by microscopy (34). To measure DNA, cells were lysed in Spheroid Lysis Buffer (Scivax, Kawasaki, Japan), and IL-1b cytokine mRNA level was measured by qRT-PCR (SuperScript IV VILO Master Mix, Waltham, MA, USA) and the 3D organoid assay was described previously $(28,29)$.

Statistical analysis. Each assay was performed in triplicate and independently repeated at least three times. The results are presented as mean \pm standard error of mean (SEM). Statistical analyses were performed using GraphPad Prism software (version 4.02; GraphPad Software, San Diego, CA, USA). One-way analysis of variance (ANOVA) followed by T-test was used to compare the results. A difference was considered significant if $p<0.05$. Statistical significance was defined as $p<0.05(*), p<0.01(* *)$, and $p<0.001(* *)$.

\section{Results}

Inflammation and metastasis-related genes in TNBC are characteristically different than genes expressed in hormonal positive breast cancer $(H R+B C)$. We first hypothesized that the subtypes of breast cancer could be distinguished by differences in gene expression. To test this idea, 53,805 
genes were assessed and their expression levels in three different hormonal positive breast cancer cell lines, ZR-75B, MCF7, and T47D, and three TNBC cell lines, MDA-MB435, MDA-MB-231, and HS578T through the National Center for Biotechnology Information (NCBI) database (Figure 1). Gene expression first underwent normalization with $\beta$-actin levels and then subjected to log-2 transformation; values greater than 1 were considered to indicate high expression while values lower than 1 indicate low expression. Genes whose expression could not be calculated or quantified are shown in black. According to the heat map shown in Figure 1, 3,728 genes were expressed in $\mathrm{HR}+\mathrm{BC}$ and 1,470 genes in TNBC (Figure 2A). Of these, 1,150 genes were co-expressed in both types of breast cancer. The DAVID database program was used to find and group the functions of each gene (Figure 2A) and the 26 genes found to be highly expressed in all three hormonal positive breast cancer cell lines correlated to cell metabolism, neuronal, and cell proliferation (Figure 1 and Figure 2B). Meanwhile, 48 genes that were highly expressed in TNBC were correlated with cell metastasis and invasion, and inflammation (Figure 1 and Figure 2C). As a result of this analysis, many genes that were related to cell metabolism, nerve-related, and cell proliferation appeared to be expressed in $\mathrm{HR}+\mathrm{BC}$, whereas cancer metastasis- and inflammation-related genes were overexpressed in TNBC. Collectively, these data indicate that TNBC has gene expression profile that is different from that of $\mathrm{HR}+\mathrm{BC}$.

Inflammation-related genes are positively related with $C d c 20$ in $T N B C$. In a previous study (2), CDC20 was found to be highly expressed specifically in TNBC through database analysis, similar to Figure 1. Breast cancer patients with high CDC20 expression showed poor overall, distant metastasisfree, and relapse-free survival $(2,22)$. Furthermore, Cdc20 was highly expressed in five different TNBC cell lines and knockdown of CDC20 completely blocked cancer cell growth and invasion in TNBC (2). We found inflammationrelated genes such as A20/TNFAIP3, CASP1, and $I L-1 \beta$, which were highly expressed in only TNBC (Figure 1). To confirm the correlation between these inflammation-related genes and $C D C 20$, we used the Breast Cancer GeneExpression Miner v4.5 (2) with 4,713 human breast cancer patient samples (Figure 3). The correlation between the genes involved in estrogen receptor positive $(\mathrm{ER}+)$, estrogen receptor negative (ER-), HER2 positive (HER2+), and TNBC was investigated. A20/TNFAIP3 did not show any correlation to $C D C 20$ in ER+ and HER2+ while there was a strong positive correlation in ER- and TNBC (Figure 3A). Interestingly, the same results were found for the other inflammation related genes, which were highly expressed in TNBC such as $C A S P 1$ and $I L-1 \beta$ (Figure $3 \mathrm{~B}$ and $\mathrm{C}$ ). Furthermore, genes highly expressed in TNBC but not related to inflammation did not show a correlation to $C D C 20$ (data not shown). Collectively, these data indicate that inflammation-related genes show a positive correlation to CDC20 only in TNBC.

High CDC20, A20/TNFAIP3, or CASP1 expression is associated with poor survival in breast cancer patients. Several studies previously reported that breast cancer patients with high $C D C 20$ expression have poor prognosis $(2,22,23)$. To examine whether the identified inflammationrelated genes highly expressed in TNBC are related to breast cancer patient prognoses, we used the Breast Cancer GeneExpression Miner v4.5 containing 4,713 human breast cancer patient samples (Figure 4). High A20/TNFAIP3 expression in breast cancer patients was related to a worse overall survival compared to a low expression (Figure 4A). This was not the case for distant metastasis-free and relapse-free survival (Figure 4A). CASPI and $I L-1 \beta$ showed similar results with A20/TNFAIP3 for breast cancer patient survival (Figure 4B and $\mathrm{C}$ ). This result suggests that high expression of inflammation-related genes, such as $C D C 20$, correlates to a worse breast cancer patient survival.

A20/TNFAIP3 positively regulates the Cdc20-Caspase 1 axis in $M D A-M B-231$. Inflammation-related genes were also highly expressed at the protein level in in five TNBC cell lines including HS578T, BT-20, BT-549, MDA-MB-231, and MDA-MB-468. As a negative control, protein levels in a normal breast cell, MCF10A, and four other breast cancers, T47D, MCF-7, BT-474, and ZR-75B were explored through western blot analysis (data not shown). A20/TNFAIP3 and caspase-1 proteins were highly expressed in all five TNBC cell lines. This agrees with our previously reported study regarding Cdc20 protein expression in TNBC cell lines (2). Based on our results, we hypothesized that A20/TNFAIP3, as a deubiquitylase $(4,10,15,16,18)$, might regulate the levels of Cdc20. To determine the signaling pathway, expression of A20/TNFAIP3, caspase-1, and Cdc20 proteins following transfection with CDC20, A20/TNFAIP3, or CASP 1 shRNA vectors individually in MDA-MB-231 was examined. Loss of A20/TNFAIP3 decreased Cdc20 and caspase- 1 protein levels, whereas $C D C 20$ knockdown only decreased caspase-1 protein levels (data not shown). However, the deletion of CASPI did not affect the protein levels of Cdc20 or A20/TNFAIP3 (data not shown), suggesting that the A20/TNFAIP3-Cdc20-Caspase-1 axis plays a role in TNBC.

A20/TNFAIP3-Cdc20-caspase-1 axis promotes the metastasis of aggressive breast cancer cells. Previous studies suggested that $\mathrm{Cdc} 20$ promoted the migration and invasion of TNBC cells (2). To determine the correlation between the A20/TNFAIP3-Cdc20-caspase-1 axis and TNBC cell 
migration and invasion, the effect of the knockdown of each A20/TNFAIP3, CDC20, or CASP1 was examined using wound healing and Boyden chamber assays (Figure 5A and C). A20/TNFAIP3 knockdown significantly decreased the migration rate of MDA-MB-231 cells compared to cells transfected with control shRNA (Figure 5B). Similar results were obtained following deletion of $C D C 20$ and $C A S P 1$ (Figure 5B). Interestingly, the effect of transfection with A20/TNFAIP3 shRNA in TNBC cells was rescued by CDC20 restitution but that of $C A S P 1$ shRNA was not (Figure 5B), suggesting that Cdc20 is regulated by A20/TNFAIP3, and caspase- 1 is downstream of Cdc20. Noticeably, CDC20 restitution did not rescue completely the effects of A20/TNFAIP3 knockdown in TNBC cells as there might be other A20/TNFAIP3 targeted molecules that influence cancer cell migration in comparison to the control shRNA (Figure $5 \mathrm{~B}$ ), demonstrating that $\mathrm{Cdc} 20$ is a downstream protein that is regulated by A20/TNFAIP3. These data are in agreement with those obtained by western blot (data not shown). Furthermore, we studied the invasion of TNBC through the Boyden chamber assay and gathered data similar to those obtained by migration assay (Figure 5D). These results suggest that A20/TNFAIP3-Cdc20-Caspase-1 axis regulates TNBC cell motility and metastasis.

Inflammatory cytokines are controlled though the A20/ TNFAIP3-Cdc20-caspase-1 axis in TNBC. Inflammationrelated cytokines have been reported to affect cancer cell migration and invasion (7-11). Therefore, the correlation between the A20/TNFAIP3-Cdc20-caspase- 1 axis and inflammation-related cytokines genes, $T N F-\alpha, I L-1 \beta$, and $I L-6$ was assessed by qRT-PCR in MDA-MB-231. In Figure 6A, $T N F-\alpha$ gene levels were significantly decreased in $C D C 20$ knockdown MDA-MB-231 cells. These data could also be found in another CDC2O shRNA. The same results were obtained for other inflammation-related cytokines, $I L-1 \beta$ and IL-6 (Figure 6A). To examine whether these data are cell line specific, BT-549 cells were used and similar results were obtained (Figure 6A). Since $\mathrm{Cdc} 20$ is up-regulated by A20/TNFAIP3 and controls Caspase-1, cytokine levels were examined in A20/TNFAIP3 or CASP1 knockdown cells (Figure 6B). $T N F-\alpha, I L-1 \beta$, and $I L-6$ levels decreased following transfection with A20/TNFAIP3 or CASP1 shRNA compared to the control group. Also, the levels of the inflammatory cytokines $T N F-\alpha$ and $I L-1 \beta$, which decreased following loss of $C D C 20$, were rescued by $C D C 20$ restitution but not following deletion of CASP1 (Figure 6C). This result suggests that $C D C 20$ has no effect on the induction of cellular inflammation in the Caspase-1/CASP1 gene deletion because Caspase$1 / C A S P 1$ is a direct downstream of $C D C 20$. Furthermore, the levels of inflammation-related cytokines were rescued following A20/TNFAIP3 knockdown with CDC20 restitution (Figure 6C), suggesting that, as previously mentioned,
A20/TNFAIP3 is a regulator of Cdc20. Together, these results show that the A20/TNFAIP3-Cdc20-caspase-1 axis is vital for the secretion of inflammation-related cytokines.

Inflammation inhibitors, necroptotic inhibitors, block TNBC metastasis. Recently several studies have shown that necroptosis is an inflammatory response that facilitates not only tumor progression but also cancer metastasis (7-9, 11, 35). Therefore, TNBC cell lines were treated with three necroptosis inhibitors, necrostatin-1 (RIPK1 inhibitor), Necrosulfonamide, and GW806742X (MLKL inhibitor) (7), and the tumorigenicity and metastatic potential of the TNBC cells grown as 3D organoid cultures were examined. The 3D organoid ex vivo system is an advanced culture method to study pharmacological effects. It is used very often because of its similarity to actual cancer cells compared to 2D cell culture. Furthermore, as the 3D organoid ex vivo directly uses human tissue, it has a higher accuracy in drug administration compared to animal experiments that have genetic dissimilarities with humans (26-32). TNBCs in 3D organoid cultures grow as spheroids a characteristic of cancer cells compared to the monolayer seen in the $2 \mathrm{D}$ tissue cell culture system and then the growth rate of cancer cells is quantified by counting the number of spheroids (Figure 7). The deletion of $C D C 20$ decreased spheroid numbers and this effect was also shown for other inflammation-related genes downregulated using shRNAs such as A20/TNFAIP3 and CASP1 (Figure 7A). On the other hand, the TNBC cell line exposed to the necroptosis inhibitor necrostatin-1 formed significantly fewer cancer spheroids than the cell group in which each of the inflammation-related genes were suppressed. Similar results were attained following treatments with necrosulfonamide and GW806742X. To determine the cancer migration and invasion rate, the stress actin fibers on the surface of cancer spheroids was counted (34). The numbers of actin fiber on the tumor organoids were decreased by the loss of $C D C 20$ and this pattern was also observed following deletion of the inflammation-related genes, A20/TNFAIP3 or CASP1 (Figure 7B). Similar to cancer spheroids, the necrostatin-1 decreased significantly more the number of actin fibers than the A20/TNFAIP3, CDC20, and CASP1 knockdowns (Figure 7B). The other necroptosis inhibitors Necrosulfonamide and GW806742X showed results similar to the necrostatin- 1 . The number of actin fiber formations was proportional to the size and number of spheroids. Finally, the levels of the inflammation-related cytokine $I L-1 \beta$ in TNBC cell 3D organoid cultures were examined. Transfection with A20/TNFAIP3, CDC20, or CASPI shRNA decreased IL-1 $\beta$ levels, and the three necroptosis inhibitors lowered the levels of the inflammatory cytokine compared to control (Figure 7C). These data suggest that the A20/TNFAIP3-Cdc20caspase- 1 axis plays an important role in cancer spheroid formation, actin fibers, and the levels of inflammation-related 

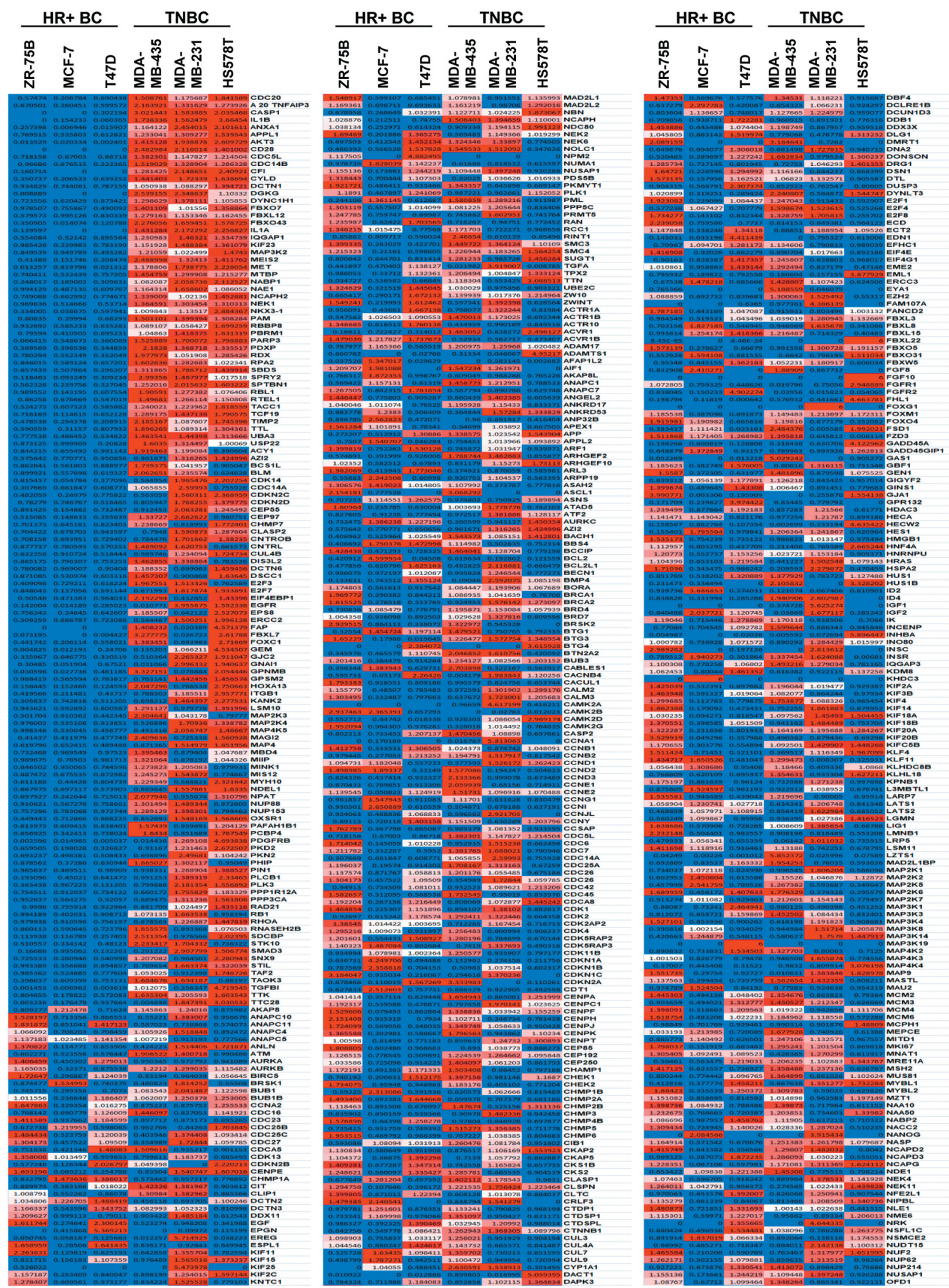

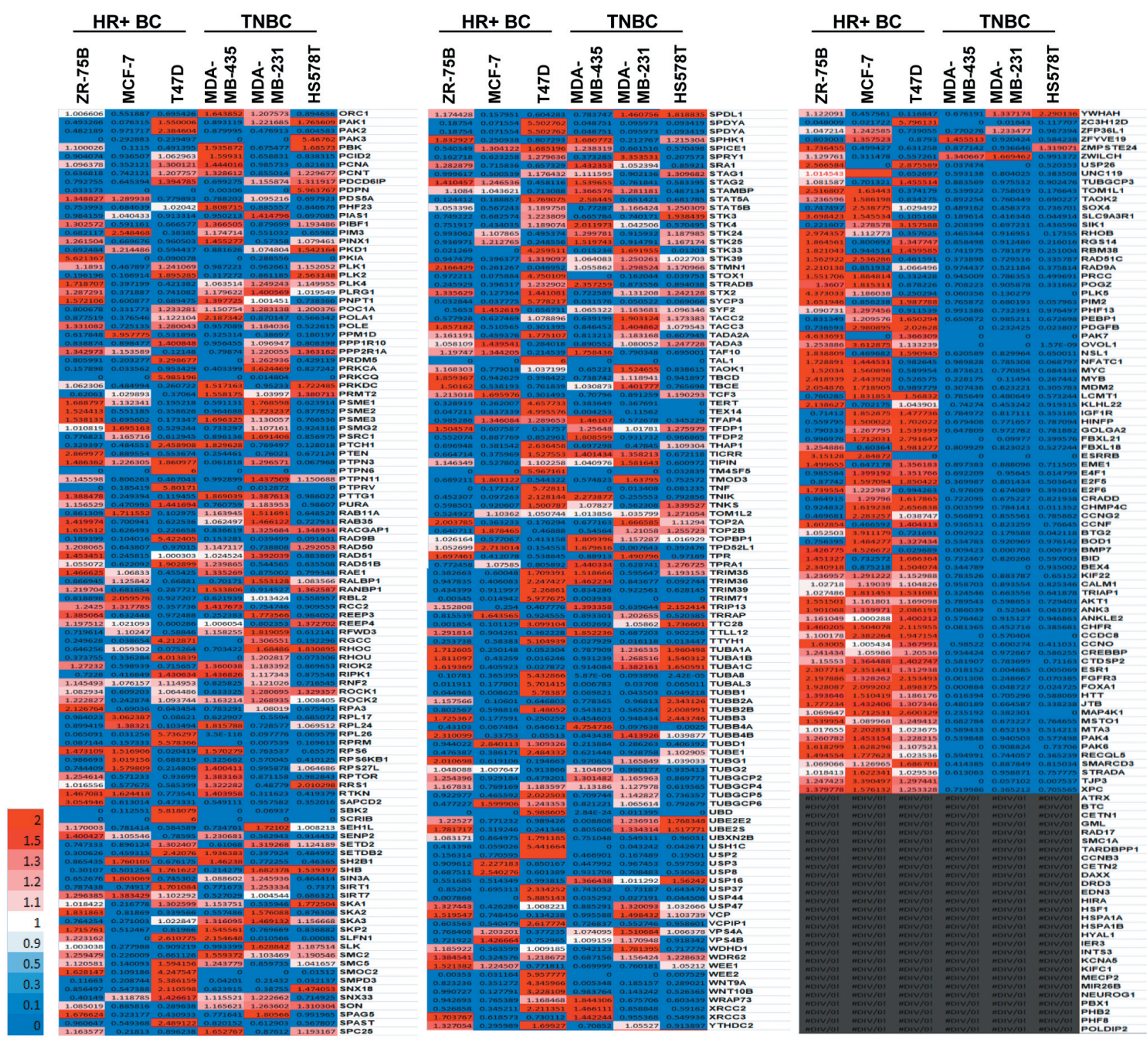

Figure 1. Inflammation and metastasis-related genes in triple negative breast cancer (TNBC) are characteristically different than genes expressed in hormonal positive breast cancer $(H R+B C)$. Heat map comparisons of differential gene expression from 53,805 genes using the Excel program. Genes that displayed greater than 1.5-fold difference $(p>0.005)$ in expression were identified and the gene expression in all six breast cancer cell lines (three TNBC; MDA-MB-231, MDA-MB-435, HS578T, and three other breast cancer; ZR-75B, MCF-7, T47D) were compared.

cytokines. Furthermore, the necroptosis inhibitors were more efficient at decreasing the TNBC cancer cell migration, invasion, and inflammatory cytokines (Figure 7D).

\section{Discussion}

Our study is the first to report that the A20/TNFAIP3-Cdc20caspase- 1 axis is highly expressed in TNBC. To examine this, a 53,805 gene database was analyzed and found the high expressions of inflammation-related genes such as
A20/TNFAIP3, CDC20, CASP1, and $I L-1 \beta$ in TNBC (Figure 1). Furthermore, these inflammatory genes associated with worse patient survival through the analysis of 4,713 patient datasets (Figure 4). We hypothesized that different groups of genes would play a critical role in different subtypes of breast cancer. In this study, we first showed that genes highly expressed in hormonal positive breast cancer correlated with cell metabolism, the nervous system, and cell proliferation, while the expression of genes related to cell invasion and inflammation was significantly higher in TNBC (Figure 2). 
A
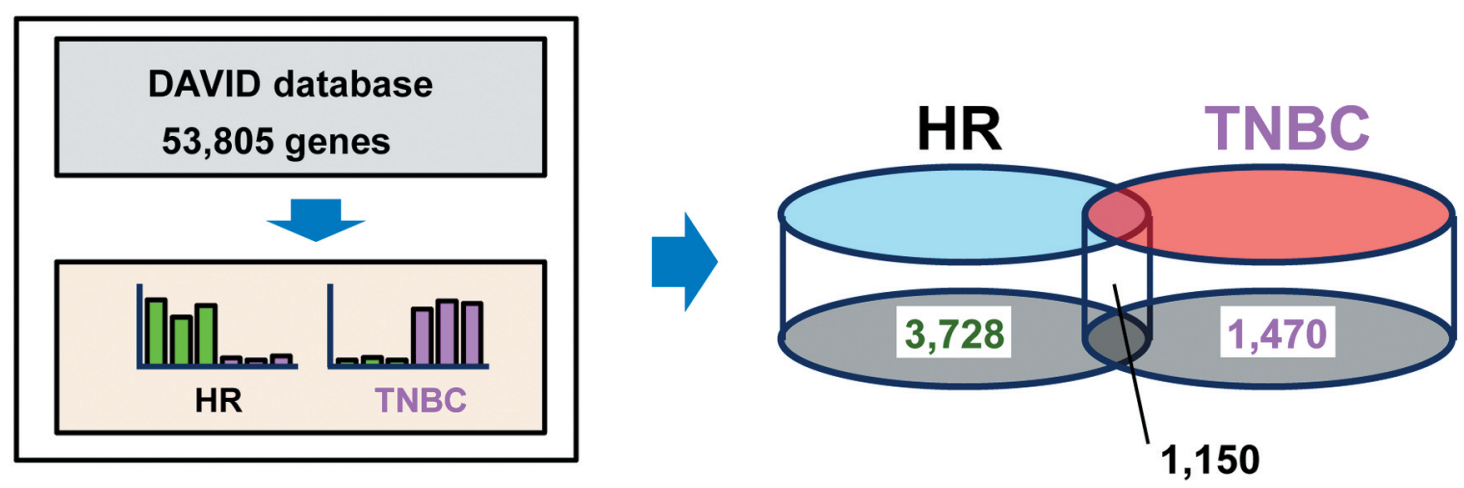

B

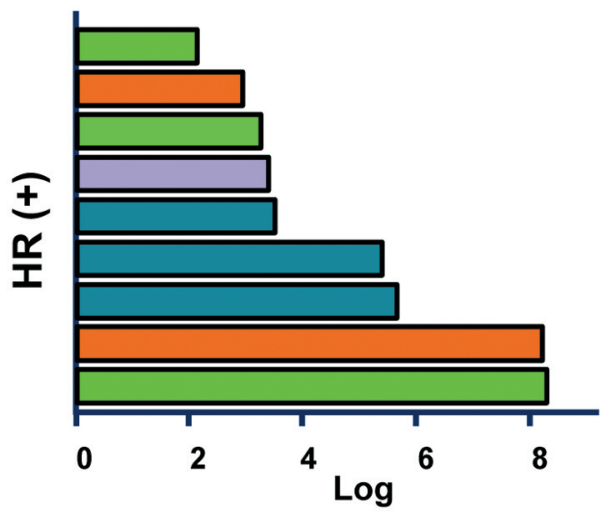

AMPK signaling pathway

Glutamatergic synapse

Mitochondria Metabolism

Peroxisome

Cell death

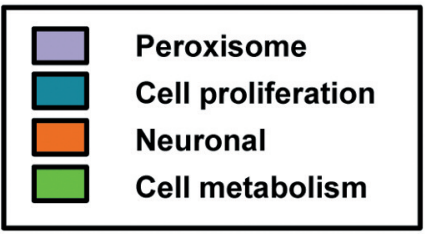

Cellular proliferation

Apoptosis

Alcoholism

Metabolic pathways

C

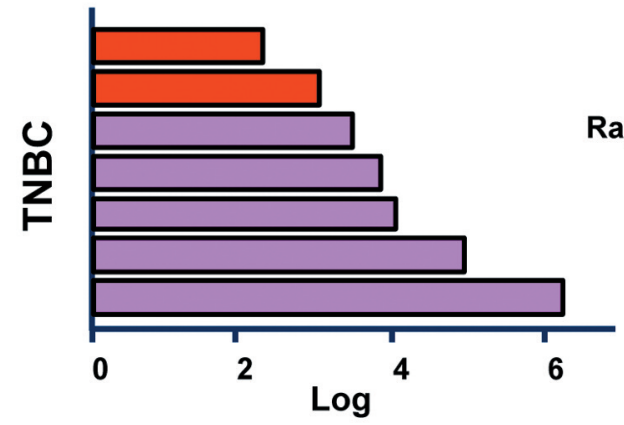

NF-kappa B signaling pathway

TNF signaling pathway

Rap1 signaling pathway (Invasion)

Pathways in cancer

Adherens junction

Metastasis and migration

\begin{tabular}{|c|}
\hline Inflammation \\
\hline $\begin{array}{l}\text { Cancer cell } \\
\text { metastasis } \\
\text { and invasion }\end{array}$ \\
\hline
\end{tabular}

(ECM and actin cytoskeleton)

Focal adhesion

Figure 2. Hormonal positive (HP) breast cancer subtype-related genes show differential expression compared to triple negative breast cancer $(T N B C)$. (A) Schematic of the overall experimental methodology and classification. (B) Characterization of genes highly expressed in HR+BC through the DAVID database. A total of 26 genes were highly expressed in all three HR+BC cell lines, which correlated to cell metabolism, neuronal, and cell proliferation. (C) Characterization of genes highly expressed in TNBC through the DAVID database. A total of 48 genes highly expressed in TNBC were correlated with cell metastasis, invasion, and inflammation. Data related to Figure 1.

Interestingly, while it was previously reported that TNBC related genes are related to cell invasion and migration, this is the first report of the correlation between TNBC and inflammation. In previous studies, we reported that high $C D C 20$ gene expression was correlated with worse patient survival, and knockdown of $C D C 20$ suppressed tumor cell growth and cancer metastasis of TNBC (2). Cdc20 also showed strong protein expression in five different TNBC cell lines, suggesting that $\mathrm{Cdc} 20$ has a very important function in tumor progression and invasion of TNBC (2). Therefore, the correlation between the inflammation-related genes found in this study and the CDC20 gene was assessed (Figure 3). This 

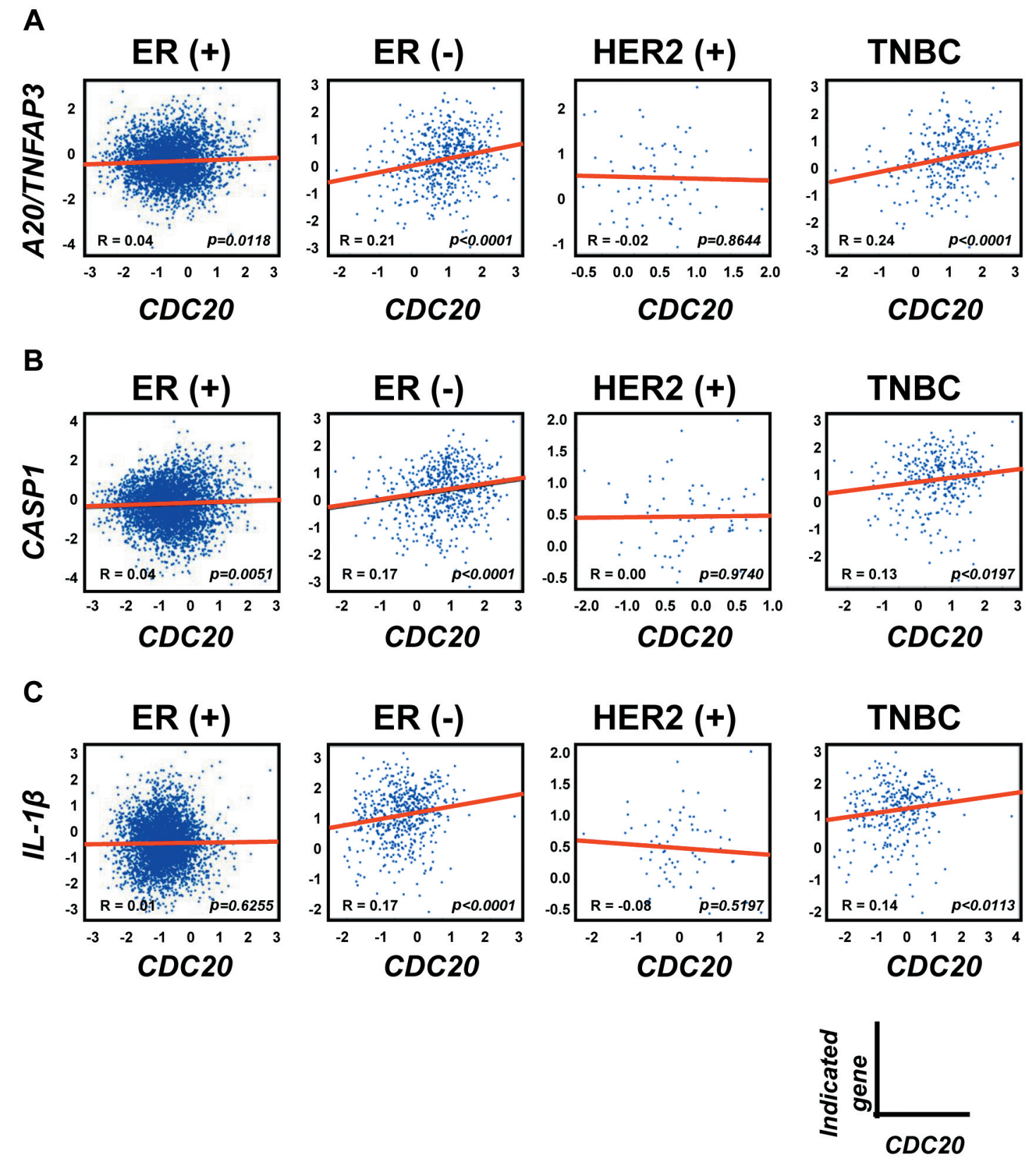

Figure 3. Inflammation-related genes are positively related with $C d c 20$ in triple negative breast cancer (TNBC). (A, B, C) Correlation plots of the score of the expression of the A20/TNFAIP3, CSAP1 and IL-1 $\beta$ with CDC20 genes in the TCGA and METABRIC cohorts. The expression of A20/TNFAIP3 and CSAP1 are positively correlated with Cdc20 especially in TNBC. Population and the event database of 4, 713 samples of patients with breast cancer patients. The p-value was calculated by Spearman's rank correlation coefficient.

study demonstrated that $C D C 20$ expression was correlated with the inflammation-related genes A20/TNFAIP3 and CASP1. High expression of A20/TNFAIP3 and CASP1 genes was associated with a poor prognosis of breast cancer patients as the survival rates significantly decreased (Figure 4). Another important finding is the new role of A20/TNFAIP3 in TNBC. A20/TNFAIP3 has been discussed in many previous studies as an anti-inflammation-related gene $(4,10,15-18)$. However, A20/TNFAIP3 acts as an anti-tumor agent in B-cell lymphoma, colorectal carcinoma, and hepatocellular carcinoma, but also as an oncogene in breast cancer, gastric cancer, and melanoma $(4,10,15,16,18)$. The present data show that high expression of the A20/TNFAIP3 gene was associated with a worse overall, distant metastasis-free, and 
A
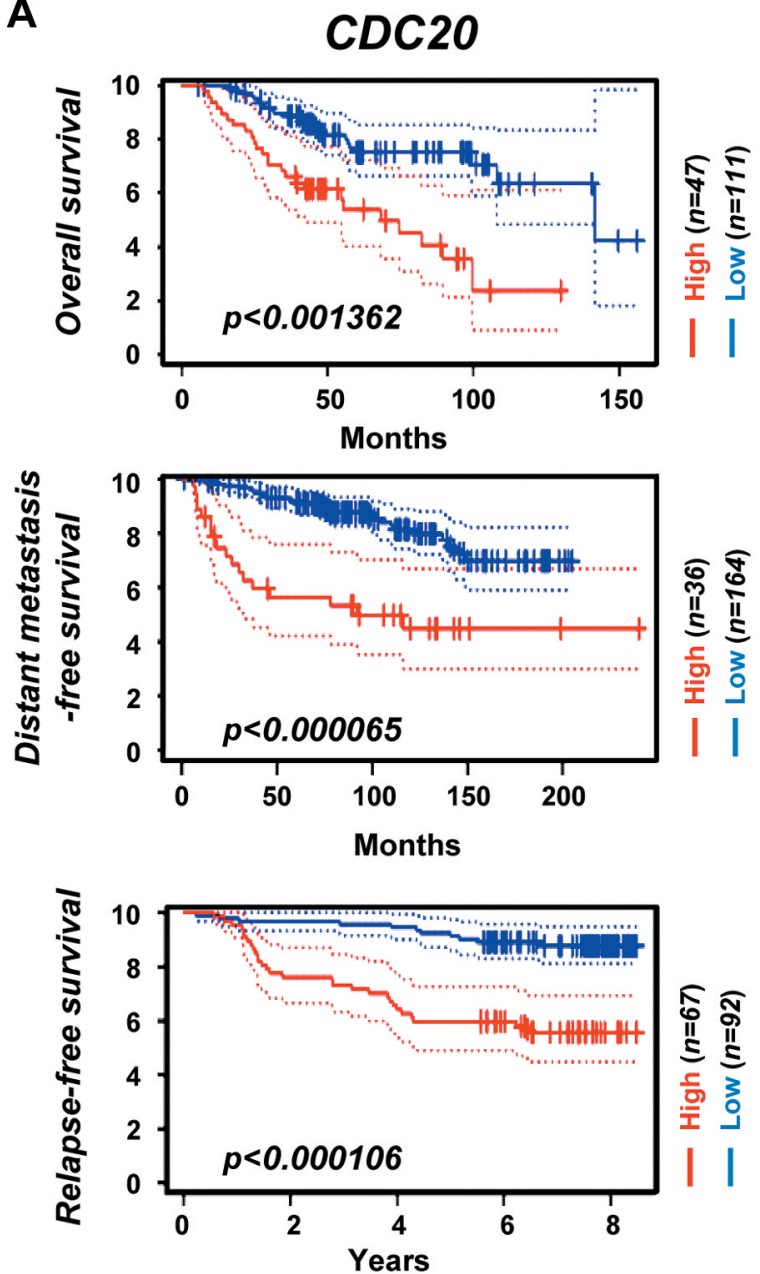

C

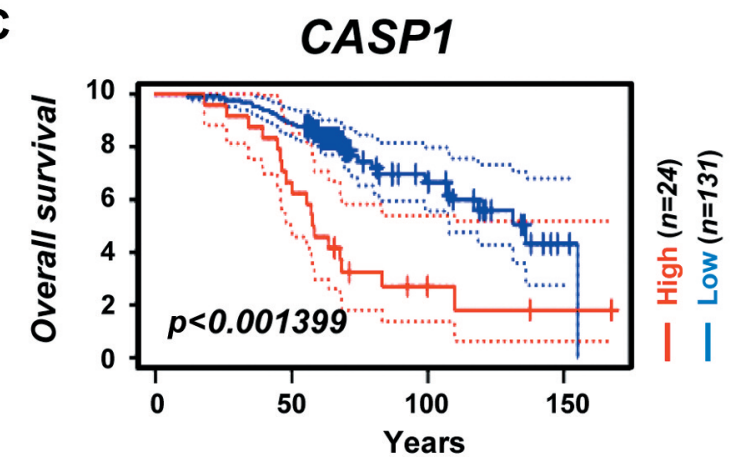

B
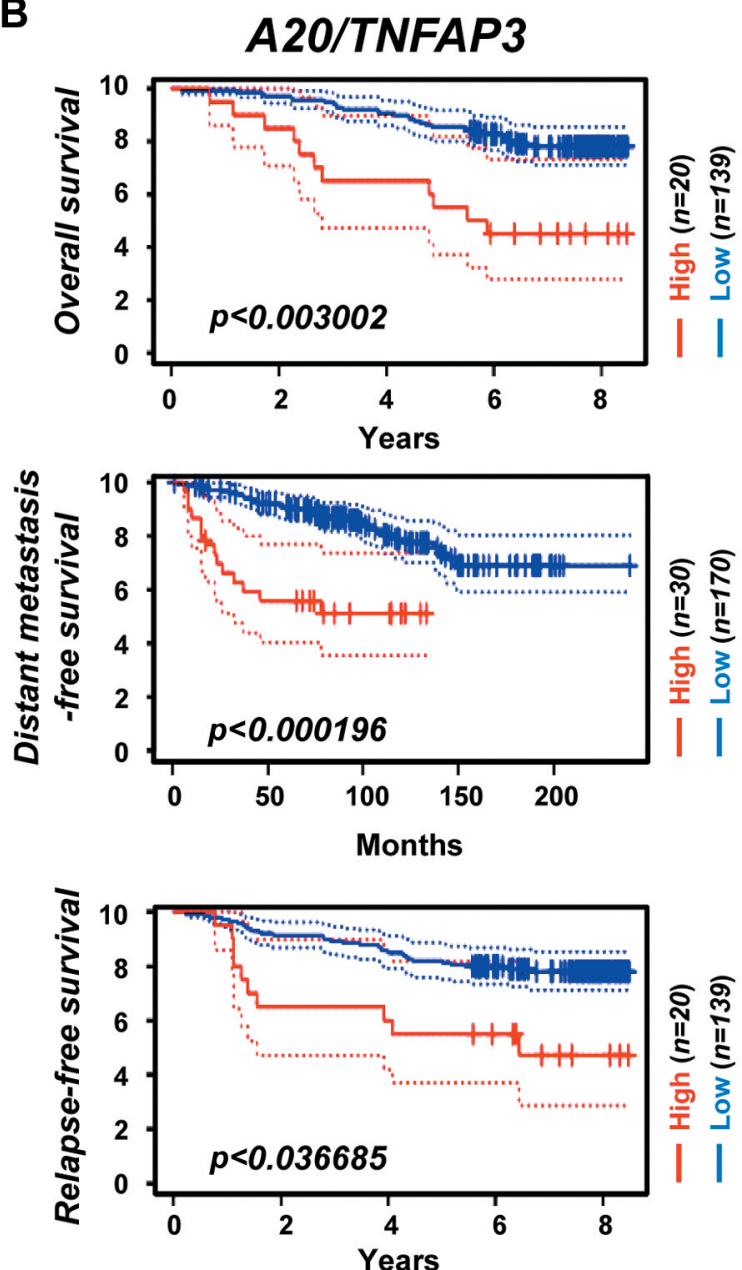

Figure 4. High CDC20, A20/TNFAIP3, and CASP1 expression is associated with poor clinical outcomes. (A, B and C) Correlation between CDC20, A20/TNFAIP3, and CASP1 expressions and clinical outcomes of breast cancer patients through the PrognoScan database (http://dna00.bio.kyutech.ac.jp/PrognoScan/index.html). A Kaplan-Meier plot analysis of overall survival (top), distant metastasis-free survival (middle), and relapse-free survival (bottom). The median values of the acquired data were used.p-Values were calculated using unpaired, two-tailed Student's t-tests. Red bar indicates high gene expression and blue bar indicates low gene expression. (GSE3143; $n=158, G S E 11121 ; n=200$, GSE1456-GPL96; $n=159$, and GSE9893; $n=155$ ).

relapse-free survival (Figure 4), and A20/TNFAIP3 protein expression was high in five different TNBC cell lines (2). Furthermore, deletion of the A20/TNFAIP3 gene markedly reduced the expression of the inflammation-related genes $T N F-\alpha, I L-1 \beta$, and $I L-6$ in TNBC (Figure 6C and Figure 7C). This suggests that A20/TNFAIP3, at least in TNBC, is likely 
A

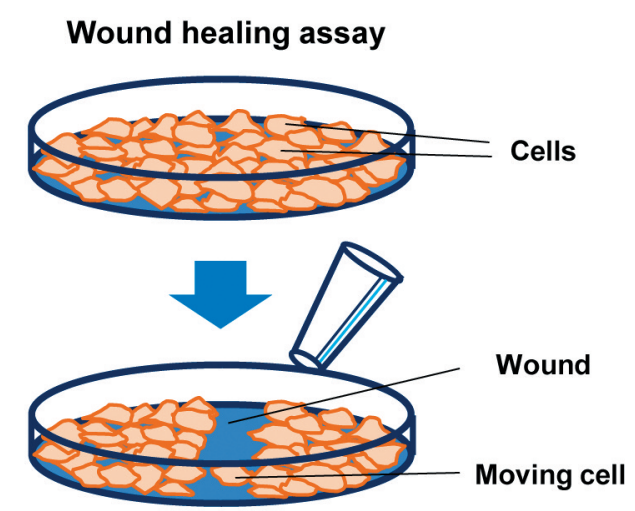

C

Boyden Chamber Assay

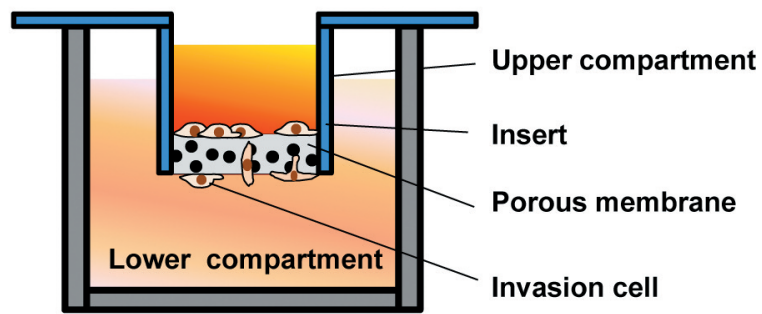

B

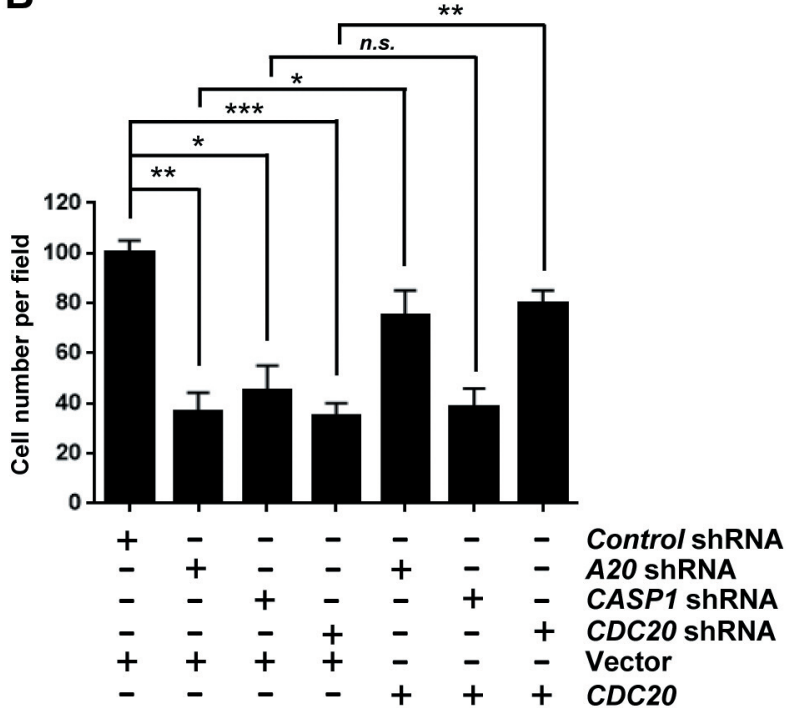

D

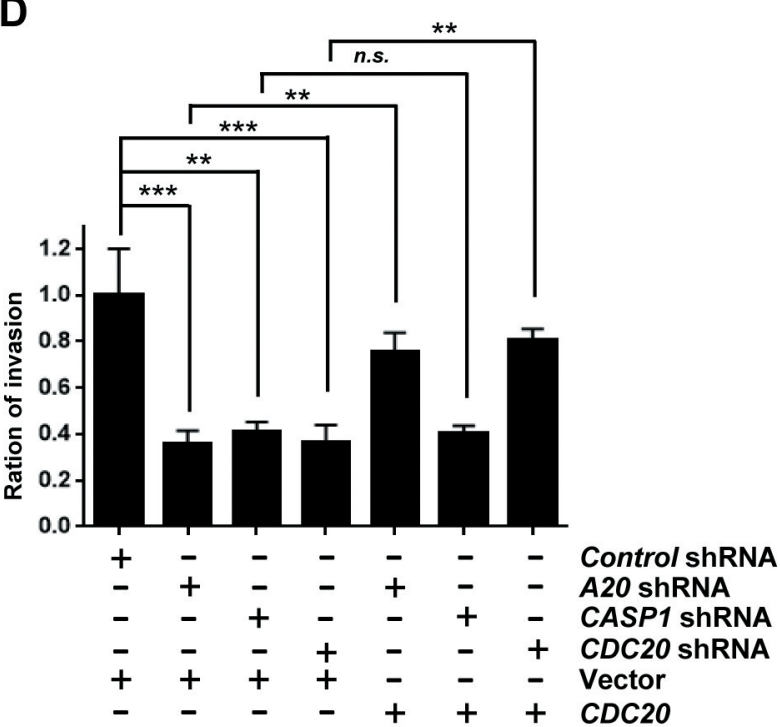

Figure 5. The A20/TNFAIP3-Cdc20-Caspase-1 axis promotes the metastasis of aggressive breast cancer cells. (A) Schematic of the overall experimental method for wound healing assay. (B) For the wound healing assay, MBA-MD-231 cells were transfected with the indicated constructs. Cell migration was assessed by the recovery of the scratch. The results are expressed as fold change relative to the corresponding blank control. Data are the means of three measurements and the bars represented $S D$ of the mean. $* p<0.05, * * p<0.01, * * * p<0.001$ by one-way ANOVA, as compared control. n.s.; no significance. (C) Schematic of the overall experimental method for Boyden chamber assay. (D) To apply the Boyden chamber invasion assay, MBA-MD-231 cells were transfected with the indicated constructs and cultured in serum free Boyden chamber for 24 h. Quantification of cell numbers results represent the means $\left( \pm\right.$ S.E.) of three independent experiments performed in triplicate. ${ }^{* *} p<0.01,{ }^{*} * * p<0.001$ by one-way ANOVA; n.s.: no significance.

a modulator of pro-inflammatory response. Therefore, more mechanistic studies are needed to elucidate the function of A20/TNFAIP3. A20/TNFAIP3 is a ubiquitin editing enzyme with both E3 ubiquitin ligase function and deubiquitinating enzyme (DUB) activity $(4,10,15,17,18)$. A20/TNFAIP3 protein consists of a specific domain related to the ovarian tumor (OTU) at the amino-terminal and seven repetitive zinc finger $(\mathrm{ZnF})$ domains at the carboxy-terminal $(4,10,16,18)$. In particular, the OTU domain is known to function as a deubiquitination (DUB) enzyme that removes the polyubiquitin chain linked to activated lysine-63 (K63) of the receptor-interacting protein kinase (RIPK), which is an essential mediator of the proximal TNFR1 complex $(4,10$, 17). In this study, the protein levels of Cdc20 were decreased upon knockdown of the A20/TNFAIP3 gene, suggesting that A20/TNFAIP3 functions as a deubiquitination (DUB) enzyme that stabilizes the substrate protein.

In this study, we used three different necroptosis inhibitors as inflammation inhibitors such as Necrostatin-1, a RIPK1 inhibitor, Necrosulfonamide, and GW806742X, an MLKL 
A
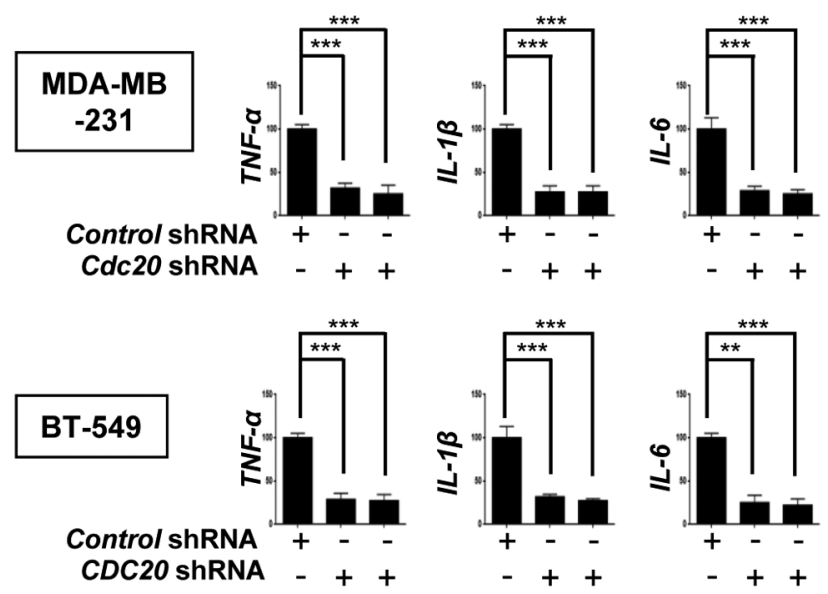

B
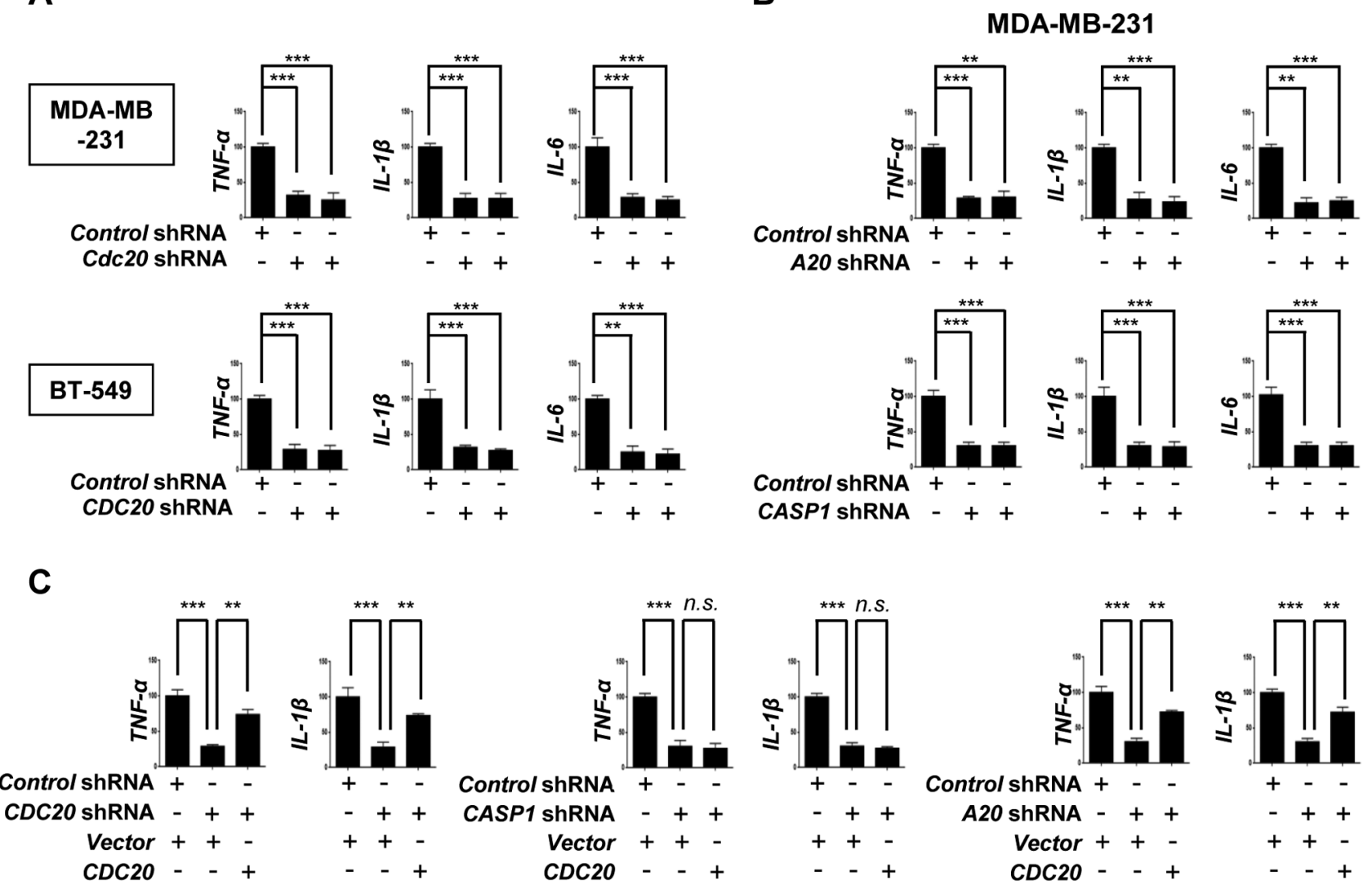

Figure 6. Inflammatory cytokines are controlled by the A20/TNFAIP3-Cdc20-Caspase-1 axis in TNBC. A, B, MDA-MB-231 or BT-549 cells were transfected with the indicated constructs. Total RNAs were isolated from transfected cells and subjected to quantitative real-time PCR ( $q R T$-PCR) measurement. The cell numbers are shown as the mean $( \pm$ S.E.) of three independent experiments performed in triplicate. $* * p<0.01$, $* * * p<0.001$ by one-way ANOVA. n.s.: not significant. (C) MDA-MB-231 cells were transfected with the indicated constructs. Total RNAs were isolated from transfected cells and subjected to real-time PCR. The cell numbers are shown as the means ( \pm S.E.) of three independent experiments performed in triplicate. $* * p<0.01$, ***p<0.001 by one-way ANOVA; n.s.: not significant.

inhibitor (Figure 7). Necroptosis is an inflammatory cell death process that is controlled mainly by Receptor-Interacting Protein Kinase 1 (RIPK1), RIPK3, and Mixed Lineage Kinase Domain-Like (MLKL). It has been reported that the main regulators of necroptosis are highly expressed in cancers $(7$, $8,35)$ and that the close relationship between necroptosis and inflammation $(7-9,11)$. Therefore, the inflammation inhibitors were used to determine whether TNBC cancer metastasis was inhibited based on a recent study on. Several studies reported that necroptosis may promote tumorigenesis and cancer metastasis due to the induction of an inflammatory response rather than a strong adaptive immune response that inhibits tumor progression $(7-9,11,35)$. The resulting necroptosis may also generate an immunosuppressive tumor microenvironment $(7,35)$. Therefore, necroptosis inhibitors were considered as potent anticancer candidates in TNBC. Interestingly, these inflammation inhibitors significantly decreased TNBC spheroid formation and actin fibers in a $3 \mathrm{D}$ organoid tissue culture system, indicating that the inflammation inhibitors prevent TNBC cell growth and metastasis. Those necroptosis inhibitors also decreased the secretion of IL-1 $\beta$ protein, suggesting that necroptosis controls cancer progression and inflammation-related cytokines.

In the future, we plan to study which genes among the A20/TNFAIP3-Cdc20-caspase-1 axis is targeted by the necroptosis-associated inflammatory inhibitors. Also, the correlation between the A20/TNFAIP3-Cdc20-caspase-1 axis and the RIPK1-RIPK3-MLKL complex, necrosome, will be also examined in the future. Although the A20/TNFAIP3 deubiquitinating enzyme (DUB) is important for the regulation of $\mathrm{Cdc} 20$, the mechanism of regulation of the necrosome is also interesting. Finally, the expression of RIPK1, RIPK3, and MLKL at the gene and protein levels in TNBC and their mechanisms also requires further study. 
A

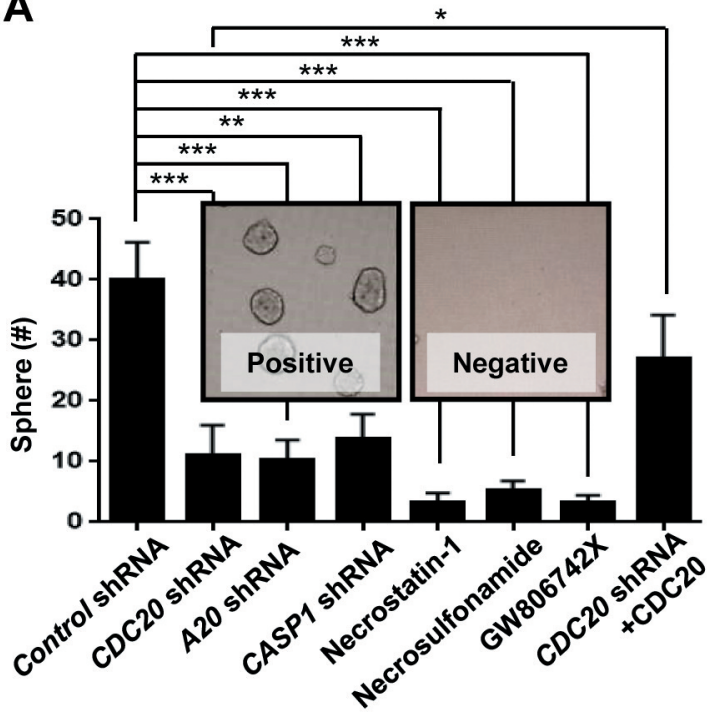

C

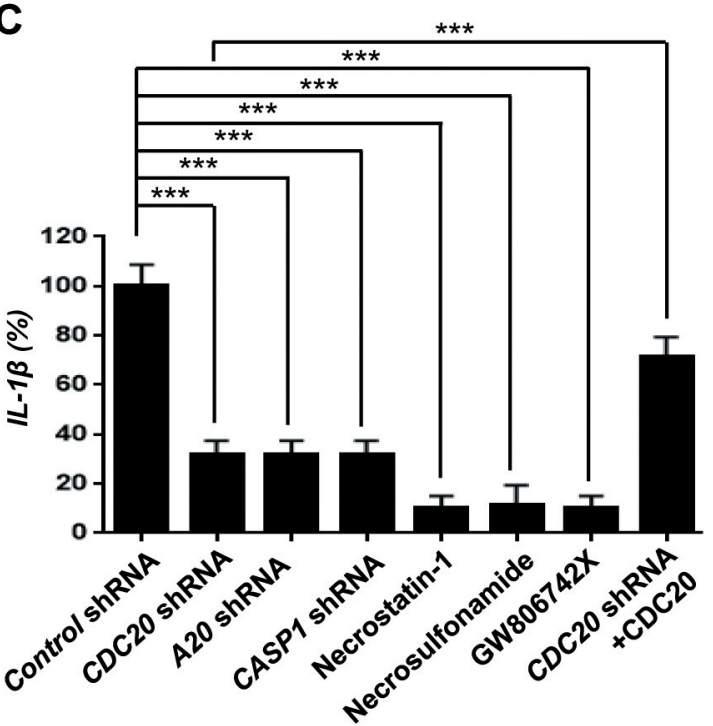

B

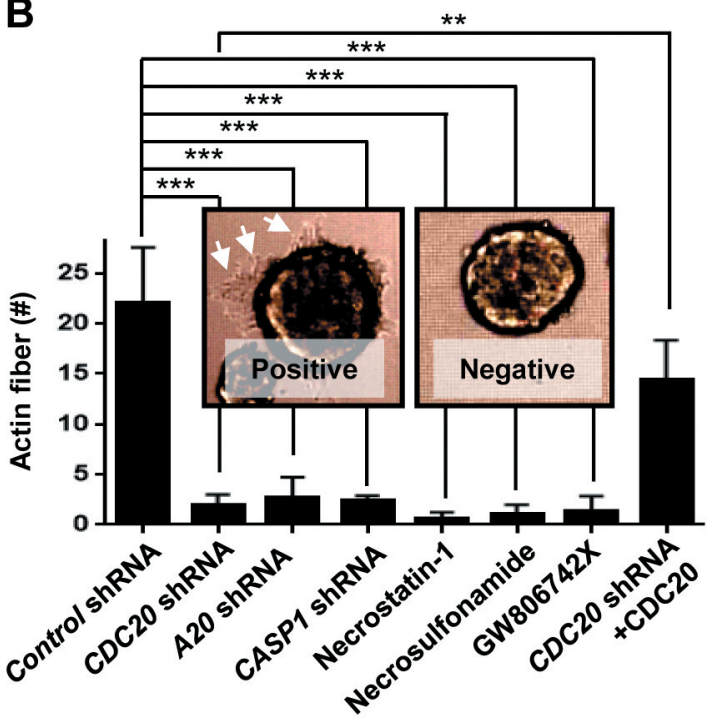

D

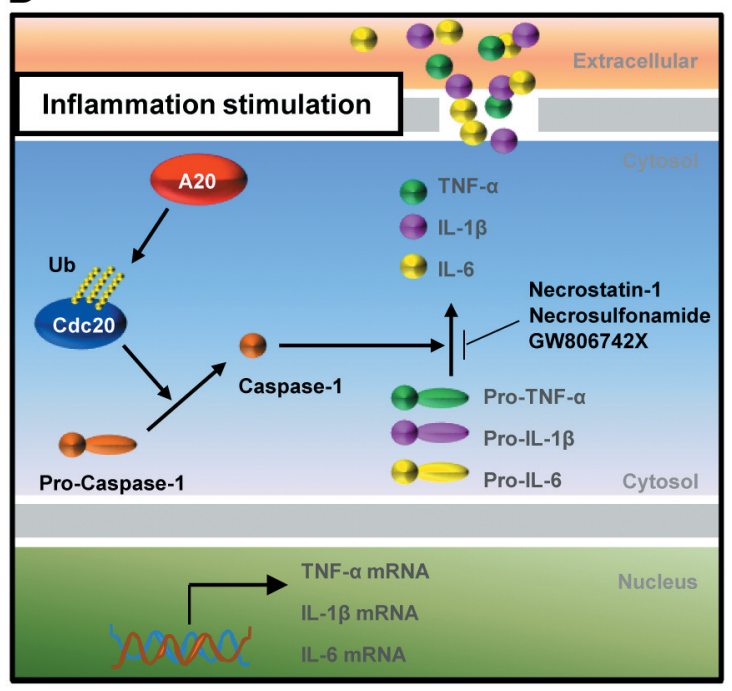

Figure 7. Inflammation inhibitors, necroptotic inhibitors, block the metastasis of TNBC. (A, B, and C) For 3D organoid ex vivo assays, MBA-MD231 cells were cultured on NanoCulture plates. After seeding for 1 day, cells were transfected with shRNAs or treated with three inflammation inhibitors, necrostatin-1 (Nec-1, $50 \mu M)$, necrosulfonamide (NSA, $10 \mu M)$, or GW806742X (GW80, $20 \mu M)$ for 24 h and observed after 7 days. Cell sphere (A) or actin fiber (B) numbers were observed by microscopy. Cells were lysed in Spheroid Lysis Buffer, and IL-1 $\beta$ cytokine was measured by $q R T-P C R(C)$. The cell numbers are shown as the means ( \pm S.E.) of three independent experiments performed in triplicate. ${ }^{*} p<0.05$, ${ }^{*} p<0.01$, $* * * p<0.001$ by one-way ANOVA. n.s.: no significance. (D) The working model.

\section{Conclusion}

In conclusion, our results demonstrate that the A20/TNFAIP3Cdc20-caspase-1 axis is associated with a poorer prognosis of patients with breast cancer and plays a significant role in TNBC cell invasion and inflammatory cytokine secretion. Furthermore, several necroptosis inhibitors decrease the TNBC cell proliferation, metastasis, and inflammation-related cytokine levels. Therefore, inflammation inhibitors may represent new therapeutics for TNBC patients.

\section{Conflicts of Interest}

Dr. Lowe is a consultant for AVID Radiopharmaceuticals, Eisai Co. Inc., Bayer Schering Pharma, GE Healthcare, and Merck Research, and receives research support from GE Healthcare, Siemens Molecular Imaging, AVID Radiopharmaceuticals, and NIH (NIA, NCI).

\section{Authors' Contributions}

S.B.L. and C.S. designed and performed most of the experiments, analyzed data, and prepared the manuscript as leading authors. V.L. 
and A.T.K. contributed to editing and commented on the article. V.L. and S.B.L. supervised the project.

\section{Acknowledgements}

The Authors thank the Jack Kent Cooke Foundation for providing support for experiments and data analysis. We also thank Zach Cohen (Jack Kent Cooke), Aaron Larson (Mayo High School, Rochester, MN, USA), Corinne Ehrfurth (Mayo High School, Rochester, MN, USA) and Christin Stegenga (Mayo High School, Rochester, MN, USA) and JungJin Kim (Mayo Clinic, Rochester, $\mathrm{MN}$, USA) for their contribution in this study.

\section{Funding}

This study was funded by the Jack Kent Cooke Foundation's Young Scholars Program.

\section{References}

1 Yin L, Duan JJ, Bian XW and Yu SC: Triple-negative breast cancer molecular subtyping and treatment progress. Breast Cancer Res 22(1): 61, 2020. PMID: 32517735. DOI: 10.1186/ s13058-020-01296-5

2 Song C, Lowe VJ and Lee S: Inhibition of Cdc20 suppresses the metastasis in triple negative breast cancer (TNBC). Breast Cancer 28(5): 1073-1086, 2021. PMID: 33813687. DOI: $10.1007 / \mathrm{s} 12282-021-01242-\mathrm{z}$

3 Medina MA, Oza G, Sharma A, Arriaga LG, Hernández Hernández JM, Rotello VM and Ramirez JT: Triple-negative breast cancer: a review of conventional and advanced therapeutic strategies. Int J Environ Res Public Health 17(6): 2078, 2020. PMID: 32245065. DOI: 10.3390/ijerph17062078

4 Lee JH, Jung SM, Yang KM, Bae E, Ahn SG, Park JS, Seo D, Kim M, Ha J, Lee J, Kim JH, Kim JH, Ooshima A, Park J, Shin D, Lee YS, Lee S, van Loo G, Jeong J, Kim SJ and Park SH: A20 promotes metastasis of aggressive basal-like breast cancers through multi-monoubiquitylation of Snail1. Nat Cell Biol 19(10): 1260-1273, 2017. PMID: 28892081. DOI: $10.1038 / n c b 3609$

5 Dai X, Cheng H, Bai Z and Li J: Breast cancer cell line classification and its relevance with breast tumor subtyping. J Cancer 8(16): 3131-3141, 2017. PMID: 29158785. DOI: $10.7150 /$ jca. 18457

6 Parise CA and Caggiano V: Breast cancer survival defined by the ER/PR/HER2 subtypes and a surrogate classification according to tumor grade and immunohistochemical biomarkers. J Cancer Epidemiol 2014: 469251, 2014. PMID: 24955090. DOI: $10.1155 / 2014 / 469251$

7 Lee SB, Kim JJ, Han SA, Fan Y, Guo LS, Aziz K, Nowsheen S, Kim SS, Park SY, Luo Q, Chung JO, Choi SI, Aziz A, Yin P, Tong SY, Fiesel FC, Springer W, Zhang JS and Lou Z: The AMPK-Parkin axis negatively regulates necroptosis and tumorigenesis by inhibiting the necrosome. Nat Cell Biol 21(8): 940-951, 2019. PMID: 31358971. DOI: 10.1038/s41556-0190356-8

8 Gong Y, Fan Z, Luo G, Yang C, Huang Q, Fan K, Cheng H, Jin $\mathrm{K}, \mathrm{Ni} \mathrm{Q}, \mathrm{Yu} \mathrm{X}$ and Liu C: The role of necroptosis in cancer biology and therapy. Mol Cancer 18(1): 100, 2019. PMID: 31122251. DOI: 10.1186/s12943-019-1029-8
9 Coussens LM and Werb Z: Inflammation and cancer. Nature 420(6917): 860-867, 2002. PMID: 12490959. DOI: 10.1038/ nature01322

10 Shi Y, Wang X, Wang J, Wang X, Zhou H and Zhang L: The dual roles of A20 in cancer. Cancer Lett 511: 26-35, 2021. PMID: 33933552. DOI: 10.1016/j.canlet.2021.04.017

11 Zhao H, Wu L, Yan G, Chen Y, Zhou M, Wu Y and Li Y: Inflammation and tumor progression: signaling pathways and targeted intervention. Signal Transduct Target Ther 6(1): 263, 2021. PMID: 34248142. DOI: 10.1038/s41392-021-00658-5

12 Sansonetti PJ, Phalipon A, Arondel J, Thirumalai K, Banerjee S, Akira S, Takeda K and Zychlinsky A: Caspase-1 activation of IL-1beta and IL-18 are essential for Shigella flexneri-induced inflammation. Immunity 12(5): 581-590, 2000. PMID: 10843390. DOI: 10.1016/s1074-7613(00)80209-5

13 Raupach B, Peuschel SK, Monack DM and Zychlinsky A: Caspase-1-mediated activation of interleukin-1beta (IL-1beta) and IL-18 contributes to innate immune defenses against Salmonella enterica serovar Typhimurium infection. Infect Immun 74(8): 4922-4926, 2006. PMID: 16861683. DOI: 10.1128/IAI.00417-06

14 Lalor SJ, Dungan LS, Sutton CE, Basdeo SA, Fletcher JM and Mills KH: Caspase-1-processed cytokines IL-1beta and IL-18 promote IL-17 production by gammadelta and CD4 T cells that mediate autoimmunity. J Immunol 186(10): 5738-5748, 2011. PMID: 21471445. DOI: 10.4049/jimmunol.1003597

15 Dong B, Lv G, Wang Q, Wei F, Bellail AC, Hao C and Wang G: Targeting A20 enhances TRAIL-induced apoptosis in hepatocellular carcinoma cells. Biochem Biophys Res Commun 418(2): 433-438, 2012. PMID: 22285182. DOI: 10.1016/j.bbrc.2012.01.056

16 Guo Q, Dong H, Liu X, Wang C, Liu N, Zhang J, Li B, Cao W, Ding T, Yang Z and Zhang X: A20 is overexpressed in glioma cells and may serve as a potential therapeutic target. Expert Opin Ther Targets 13(7): 733-741, 2009. PMID: 19492975. DOI: $10.1517 / 14728220903045018$

17 Vendrell JA, Ghayad S, Ben-Larbi S, Dumontet C, Mechti N and Cohen PA: A20/TNFAIP3, a new estrogen-regulated gene that confers tamoxifen resistance in breast cancer cells. Oncogene 26(32): 4656-4667, 2007. PMID: 17297453. DOI: 10.1038/ sj.onc. 1210269

18 Chen S, Xing H, Li S, Yu J, Li H, Liu S, Tian Z, Tang K, Rao Q, Wang $M$ and Wang J: Up-regulated A20 promotes proliferation, regulates cell cycle progression and induces chemotherapy resistance of acute lymphoblastic leukemia cells. Leuk Res 39(9): 976-983, 2015. PMID: 26159495. DOI: 10.1016/j.leukres.2015.06.004

19 Lee SB, Kim JJ, Nam HJ, Gao B, Yin P, Qin B, Yi SY, Ham H, Evans D, Kim SH, Zhang J, Deng M, Liu T, Zhang H, Billadeau DD, Wang L, Giaime E, Shen J, Pang YP, Jen J, van Deursen $\mathrm{JM}$ and Lou Z: Parkin regulates mitosis and genomic stability through Cdc20/Cdh1. Mol Cell 60(1): 21-34, 2015. PMID: 26387737. DOI: 10.1016/j.molcel.2015.08.011

$20 \mathrm{Yu} \mathrm{H}$ : Cdc20: a WD40 activator for a cell cycle degradation machine. Mol Cell 27(1): 3-16, 2007. PMID: 17612486. DOI: 10.1016/j.molcel.2007.06.009

21 Kapanidou M, Curtis NL and Bolanos-Garcia VM: Cdc20: At the crossroads between chromosome segregation and mitotic exit. Trends Biochem Sci 42(3): 193-205, 2017. PMID: 28202332. DOI: 10.1016/j.tibs.2016.12.001

22 Karra H, Repo H, Ahonen I, Löyttyniemi E, Pitkänen R, Lintunen M, Kuopio T, Söderström M and Kronqvist P: Cdc20 
and securin overexpression predict short-term breast cancer survival. Br J Cancer 110(12): 2905-2913, 2014. PMID: 24853182. DOI: $10.1038 /$ bjc.2014.252

23 Wang L, Zhang J, Wan L, Zhou X, Wang Z and Wei W: Targeting $\mathrm{Cdc} 20$ as a novel cancer therapeutic strategy. Pharmacol Ther 151: 141-151, 2015. PMID: 25850036. DOI: 10.1016/j.pharmthera.2015.04.002

24 Liu J, Zhang C, Hu W and Feng Z: Parkinson's diseaseassociated protein Parkin: an unusual player in cancer. Cancer Commun (Lond) 38(1): 40, 2018. PMID: 29941042. DOI: 10.1186/s40880-018-0314-Z

25 Stieg DC and Cooper KF: Parkin new cargos: a new ROS independent role for Parkin in regulating cell division. React Oxyg Species (Apex) 2(5): 315-324, 2016. PMID: 28920079. DOI: $10.20455 /$ ros.2016.857

26 Driehuis E, Kretzschmar K and Clevers H: Establishment of patient-derived cancer organoids for drug-screening applications. Nat Protoc 15(10): 3380-3409, 2020. PMID: 32929210. DOI: 10.1038/s41596-020-0379-4

27 Liu C, Qin T, Huang Y, Li Y, Chen G and Sun C: Drug screening model meets cancer organoid technology. Transl Oncol 13(11): 100840, 2020. PMID: 32822897. DOI: 10.1016/j.tranon.2020. 100840

28 Kim JJ, Lee SB, Yi SY, Han SA, Kim SH, Lee JM, Tong SY, Yin P, Gao B, Zhang J and Lou Z: WSB1 overcomes oncogene-induced senescence by targeting ATM for degradation. Cell Res 27(2): 274293, 2017. PMID: 27958289. DOI: 10.1038/cr.2016.148

29 Lee S, She J, Deng B, Kim J, de Andrade M, Na J, Sun Z, Wampfler JA, Cunningham JM, Wu Y, Limper AH, Aubry MC, Wendt C, Biterman P, Yang P and Lou Z: Multiple-level validation identifies PARK2 in the development of lung cancer and chronic obstructive pulmonary disease. Oncotarget 7(28): 44211-44223, 2016. PMID: 27329585. DOI: 10.18632/oncotarget.9954

30 Liu HD, Xia BR, Jin MZ and Lou G: Organoid of ovarian cancer: genomic analysis and drug screening. Clin Transl Oncol 22(8): 1240-1251, 2020. PMID: 31939100. DOI: 10.1007/ s12094-019-02276-8
31 Chambers KF, Mosaad EM, Russell PJ, Clements JA and Doran MR: 3D Cultures of prostate cancer cells cultured in a novel high-throughput culture platform are more resistant to chemotherapeutics compared to cells cultured in monolayer. PLoS One 9(11): e111029, 2014. PMID: 25380249. DOI: 10.1371/journal.pone.0111029

32 Bengtsson A, Andersson R, Rahm J, Ganganna K, Andersson B and Ansari D: Organoid technology for personalized pancreatic cancer therapy. Cell Oncol (Dordr) 44(2): 251-260, 2021. PMID: 33492660. DOI: 10.1007/s13402-021-00585-1

33 Kim JJ, Lee SB, Jang J, Yi SY, Kim SH, Han SA, Lee JM, Tong SY, Vincelette ND, Gao B, Yin P, Evans D, Choi DW, Qin B, Liu T, Zhang H, Deng M, Jen J, Zhang J, Wang L and Lou Z: WSB1 promotes tumor metastasis by inducing pVHL degradation. Genes Dev 29(21): 2244-2257, 2015. PMID: 26545811. DOI: $10.1101 / \operatorname{gad} .268128 .115$

34 Godinho SA, Picone R, Burute M, Dagher R, Su Y, Leung CT, Polyak K, Brugge JS, Théry M and Pellman D: Oncogene-like induction of cellular invasion from centrosome amplification. Nature 510(7503): 167-171, 2014. PMID: 24739973. DOI: 10.1038 /nature 13277

35 Wang T, Jin Y, Yang W, Zhang L, Jin X, Liu X, He Y and Li X: Necroptosis in cancer: An angel or a demon? Tumour Biol 39(6): 1010428317711539, 2017. PMID: 28651499. DOI: $10.1177 /$ 1010428317711539
Received November 27, 2021

Revised December 24, 2021

Accepted December 31, 2021 